\title{
ERRATUM
}

\section{Study on the Purification of Polysaccharides from Noscoc flagelliforme with Radial Flow Chromatography}

\author{
Yu-Jie Dai*, Jing-Wen Wang, Shi-Ru Jia, Si-Jun Yue, Meng-Yao Jia, and Peng Xu
}

Key Laboratory of Industrial Microbiology, Ministry of Education, College of Biotechnology, Tianjin University of Science and Technology, Tianjin 300-457, China

In the May/June 2009 issue of Biotechnology and Bioprocess Engineering (BBE), errors occurred in the research article:

Yu-Jie Dai, Jing-Wen Wang, Shi-Ru Jia, Si-Jun Yue, Meng-Yao Jia, and Peng Xu (2008) Study on the Purification of Polysaccharides from Noscoc flagelliforme with Radial Flow Chromatography. Biotechnol. Bioprocess Eng. 14: 377-382.

- In the Title, Abstract, and Keywords

Original wording:

Noscoc

This should be replaced by:

Nostoc

\section{- In the REFERENCES}

\section{Original wording:}

Yu, H. F., S. R. Jia, and Y. J. Dai (2008) Growth characteristics of the cyanobacterium $N$. flagelliforme in photoautotrophic, mixotrophic, and heterotrophic cultivation. J. Appl. Phycol. DOI/10.1007/s10811008-9341-5.

This should be replaced by:

Yu, H. F., S. R. Jia, Y. J. Dai (2009) Growth characteristics of the cyanobacterium Nostoc flagelliforme in photoautotrophic, mixotrophic, and heterotrophic cultivation. J. Appl. Phycol. 21:127-133.

\section{- On page 378, left column first line}

\section{Original wording:}

the growth rate per year is only $10 \%$

This should be replaced by:

the growth rate is less than $6 \%$ per year
*Corresponding author

Tel: +86-22-60601268 Fax: +86-22-60602298

e-mail: yjdai@126.com 